Aus der Neuen Heilanstalt für Lungenkranke in Schömberg O.-A. Neuenbürg.

\title{
Ueber die Behandlung der Larynx- tuberkulose').
}

Von Dr. G. Schröder, dirigierendem Arzt.

M. H.: Wenn ich es unternehnie, Ihnen meine Erfahrungen über die Behandlung der Larynxtuberkulose mitzuteilen, so veranlaßt mich dazu in erster Linie die Divergenz der Meinungen, die trotz des vorliegenden Tatsachenmaterials über Indikationen und Aussichten der Therapie dieser Organtuberkulose noch vorherrscht. Konnte doch erst kürzlich ein italienischer Kliniker den Satz aussprechen: ,Die Bemühungen der Laryngologen um die Behandlung der Kehlkopftuberkulose als eines lokalen Prozesses sind völlig erfolglos geblieben." (1) - Man sollte es nicht glauben, daß die jahrelangen Bestrebungen bekannter Laryngologen, wie M. Schmidt, Krause, Heryng, Krieg, Burger, Mernod, Blumenfeld u. a., sowie vieler Anstaltsärzte, ich nenne nul Turban, Besold, Gidionsen und mich selbst, um eine erfolgreiche Bekämpfung dieser Tuberkuloseform noch immer nicht ein abschließendes Urteil über die Art und den Nutzen ihrer Therapie gestatten, sogar noch diesen Skeptizismus des italienischen Autors möglich machen.

Ich will es vermeiden, vor Ihnen die enorme Literatur der letzten 20 Jahre über diese Fragen Revue passieren zu lassen. Es würde ermüden und zu keinem Resultat führen. Wir kommen unserer Ansicht nach eher zu einer Klärung der Auffassungen und zu einer optimistischeren Beurteilung unseres Könnens in der Therapie der Larynxtuberkulose, wenn jeder, der sich mit dieser Krankheit besonders befaßte, sein Krankenmaterial nöglichst objektiv sichtet und seine Indikationen ill der Therapie und seine Erfolge kritisch mitteilt.

In den letzten 14 Jahren beobachtete ich im ganzen 416 Fälle von Larynxtuberkulose aller Stadien und Formen. Diese Fälle wurden sämtlich in der geschlossenen Anstalt durchschnittlich 140 Tage behandelt. Ich bin daher vielleicht besser als mancher Laryngologe, der seine Kranken nur ambulant behandelt, in der Lage, über den Nutzen therapeutischer Maßnahmen zu urteilen. - Die Larynxtuberkulose ist eine der häufigsten Komplikationen der chronischen Lungentuberkulose. Wir können annehmen, daß etwa $20 \%$ unserer Kranken sie aufweisen.

Von größter Wichtigkeit ist auch bei dieser Tuberkulosefornı die Frühdiagnose. Jeder Lungentuberkulöse muls genau auf Veränderungen in den oberen Luftwegen untersucht werden; der Arzt, der das versäumt oder diese Untersuchung, wenn er sie nicht selbst vornehmen will, von einem Spezialisten nicht machen läßt, begeht eine Unterlassungssünde. Die ersten tuberkulösen Veränderungen im Larynx machen bekanntlich oft nicht die geringsten Beschwerden. Jede einseitige Rötung eines Stimmbandes, jede geringste lokalisierte Schwellung der Schleimhaut im Kehlkopfe eines Lungentuberkulösen ist als tuberkulöser Prozeß anzusehen und $\mathrm{zu}$ behandeln. Tuberkulin zur Frühdiagnose bei dieser Tuberkulosefornı anzuwenden, können wir nicht empfehlen. Herdreaktionen haben hier große Gefahren. Es kann zur Einschnielzung und Propazation des Prozesses kommen. Empfehlenswert ist, die verdächtige Schleimhaut mit KokainAdrenalinlösungen $\mathrm{zu}$ pinseln. Die Herde heben sich dann oft plastisch aus ihrer anämisierten Umgebung ab.

Unserer Ansicht nach erfordert die Larynxtuberkulose, auch schon in Anfangsstadium, stets Anstaltsbehandlung. Wie wir bei Besprechung der Therapie sehen werden, sind unsere

1) Vortrag, gehalten auf der Versammlung der Lungenheilanstalty. ärzte vom 17.- 19. September 1910 in_München. 
Maßnahmen am sichersten und erfolgversprechendsten nur in einer geschlossenen Anstalt durchzuführen, wo der Arzt den Kranken ganz in der Hand hat und auch die Ausheilung der Lunge, die ja stets miterkrankt ist, am besten gefördert werden kann.

Wie Lungen- und Halstuberkulose ätiologisch im engsten Konnex stehen, so ist auch eine Ausheilung beider Prozesse in derartigem Abhängigkeitsverhältnis, daß eine getrennte Behandlung stets eine halbe Behandlung bleibt. Wir wollen daher auch heute wieder die Forderung aufstellen, da $\beta$ siclı die Volksheilstätten auch der heilbaren Larynxphthisiker annehmen müssen.

Wir konimen jetzt zur Therapie: Unsere letzten Sätze haben es schon klar ausgesprochen, daß die allgemeine Phthiseothe:apie, d. h. da; stre -ge hygienisch-diätetische Regime, wie es in den geschlossenen Anstalten geübt wird, auch für den Larynxtuberkulösen von eminentester Bedeutung ist. Es bildet den Grundpfeiler unserer Behandlung. Die lokale Behandlung unterstiitzt sie nur wirksam. Es kommen hier zwei Methoden in Frage: 1. Die mehr konservativen Maßnahmen, 2. die chirurgisch-operativen. Hinsichtlich der Wahl der Methoden herrscht nun noch durchaus keine Einigkeit.

Um nur ein krasses Beispiel der Gegensätze anzuführen, nenne ich einen Ausspruch Pottengers (2), der gegen jede operative Maßnahme bei der Larynxtuberkulose protestiert. Andere wieder versprechen sich nur Erfolg von chirurgischem Vorgehen. Hier wendet der eine nur Aetzungen mit Säuren an, der zweite bevorzugt die Galvanokaustik, der dritte das blutige Vorgehen mit Kuretten und schneidenden Zangen. Eine strenge Indikationsstellung für das eine oder andere Verfahren vermissen wir. Auf Grund unserer Erfahrungen will ich versuchen, die Wahl der Methoden zu umgrenzen.

Für uns gilt als Richtschnur für jedes aktive, chirurgische Vorgehen bei einem Larynxtuberkulösen der Grundsatz: Es darf nur von der streng konservativen Therapie bei einem Kranken mit anormaler Temperatur abgewichen werden, wenn eine Indicatio vitalis vorliegt oder Schwellungen und Schmerzen die Nah rungsaufnahme behindern. Es muB so lange konservativ behandelt werden, bis der Kranke dauernd entfiebert ist und der Allgemeinzustand sich erheblich gebessert hat. Besold und Gidionsen (3) haben diesen Grundsatz in ihrem Buche (S. 88) auch schon angedeutet. Wir wissen aber, daß er nicht Allgemeingut der Spezialisten geworden ist. Jeder Larynxphthisiker ist demnach einer genausten Beobachtung zu unterwerfen; er muß zweistündlich gemessen werden. Es soll alles versucht werden, die Lungen zu bessern und das Allgemeinbefinden zu heben. Sind die Kräfte vermehrt, ist dauernd Afebrilität erreicht, dann haben chirurgische Eingriffe im Kehlkopf, wenn noch nötig, zu erfolgen und werden segensreich wirken. Ich sage, wenn noch nötig. Sehr oft werden wir sehen, daß der lokale Prozeß im Halse sich bei solchen Kranken schon spontan, vielleicht mit Unterstützung unserer konservativen Behandlungsmethoden, sehr zurückbildete, oft sogar zur Heilung kam. - Meine Herren, nehmen Sie sich diesen Grundsatz zur Richtschnur für Ihr Vorgehen bei der Therapie der Larynxtuberkulose, Sie werdendann weniger Mißerfolge von operativen Eingriffen erleben und nicht mutlos Kauter und Kurette von sich werfen. Die Mißerfolge so mancher bedeutender Laryngologen bei chirurgischem Vorgehen führe ich darauf zurück, daß der Allgemeinzustand der Kranken nicht genügend berücksichtigt wurde. Es wurde nicht gemessen. Die Lungen wurden nicht genügend beobachtet und kontrolliert. Die Lebensweise der Patienten wurde nicht geregelt. Der fiebernde Tuberkulöse verträgt keine energischeren Eingriffe im Halse. Seine Temperatur steigt danach oft sehr hoch, er neigt mehr zu sekundären Schwellungen. Dadurch bildet sich ein Circulus vitiosus. Die Ernährung wird erschwert, die Lunge schlechter, und das Resultat ist ein kläglicher Mißerfolg. Es ist selbstredend, daß. neben dem Fieber noch andere Komplikationen der Lungentuberkulose sehr zur Vorsicht bei Eingriffen im
Larynx ermahnen. Ich erwähne nur schwere Anämie, sehr große Unterernährung, Magen-, Darmstörungen, Darmtuberkulose, mit einem Worte Komplikationen, die eine Neigung zur Propagation des Lungenprozesses befürchten lassen. Solche Fälle sind stets erst längere Zeit zu beobachten; diese Komplikationen sind niöglichst zu bessern und zu beheben, ehe wir ohne Sorgen energischer im Kehlkopf eingreifen dürfen. Daß bei tuberkulösen Frauen während der Menses Eingriffe im Kehlkopf zu unterbleiben haben, bedarf wohl nicht der Erwähnung.

Soviel über die allgemeine Richtschnur bei der Indikationsstellung für operatives Vorgehen. Auf spezielle Indikationen für die Wahl der einen oder der anderen Operationsmethode werde ich noch bei der Besprechung der Methoden selbst zurückkommen. - Ich möchte Ihnen jetzt in kurzen Zügen unsere lokale Behandlung schildern, wie wir sie auf Grund unserer langjährigen Erfahrung iiben.

\section{Konservative Therapie.}

Dem Kranken wird völlige Schonung seines erkrankten Organs auferlegt. Er muß möglichst absolut schweigen. Der Husten ist zielbewußt zu bekänıpfen, möglichst ohne Narcotica. Die Diät sei reizlos. Alcoholica meiden wir bei Halskranken fast ganz, vor allem das Bier. Rauchen ist selbstverständlich zu verbieten. Jeder Kranke erhält nachts, wenn er bettlägerig ist auch am Tage, Halswickel, die mit Alkohol und Wasser $\overline{a a}$ gemacht werden. Bei Schwellungen hat sich ständiges Tragen der Eiskrawatte auch uns als nützlich erwiesen. In manchen Fällen leistete das Stauen Gutes (Tragenlassen eines Gummibändchens unterhalb des Pomum Adami täglich mehrere Stunden). Die Stauung schien vor allem akute Reizerscheinungen in der Schleimhaut, sekundäre Laryngitis zu mildern, Schluckbeschwerden zu bessern. Alle Begleitkatarrhe in Nase und Pharynx sind zu behandeln und möglichst zu heilen. -- Die Grundzüge der Therapie sind also neben der Allgemeinbehandlung des Kranken: Ruhigstellung des erkrankten Organs, Fernhalten von Reizungen durch die Nahrung, durch herabfließendes Sekret aus Nase und Pharynx, ev. Erzielen einer Hyperämie durch Alkoholpackungen und Stauung. Die Besonnung der erkrankten Teile, von der viele Autoren Gutes sahen, hat mir keine nennenswerte Vorteile gebracht. Wir sind mehr davon zurückgekommen, da der Nutzen dieser Therapie die mancherlei Nachteile durch Anstrengen der Kranken nicht aufwiegt.

Die konservative Lokalbehandlung umfaßt das Einbringen von flüssigen und pulverförmigen Arzneien in den Kehlkopf und die Inhalationen. Hier wird jeder Therapeut seine Lieblingsmittel haben. Wir richten uns nach der Empfindlichkeit des Organs bei den einzelnen Kranken und nach dem lokalen Befunde. Sind Vlcera vorhanden mit Reizerscheinungen in ihrer Umgebung, so leisten anästhesierende Pulver Gutes. Ich nenne nur Anästhesin und Orthoform. Fehlen Reizerscheinungen der Schleimhaut, sind Jodol und Mentholjodol zu empfehlen. Bei Infiltraten, die nicht zerfallen sind, möchte ich das Mentholöl loben in steigender Konzentration $\left(5-25^{\circ}{ }_{0}\right)$, oder mit Orthoform in $5-10 \%$ Konzentration, Eigelb und Oel emulgiert. (Rp. Orthoform, Menthol aa 2,5 $-5,0$ vitell. ovi un. Olei amygdal. dulc., Olei olivar. aa ad 50,0.) Alle diese Mittel werden täglich ein- bis zweimal in den Larynx gebracht, bei Schluckschmerzen am besten vor den Mahlzeiten. Dann kann man auch kleinste Dosen Morphin (5 mgr) mit Sacch. lact. vor dem Essen auf den Zungengrund oder auf den Aditus laryngis applizieren. Weitere Schluckschmerzen erfordern Gebrauch von Alipin oder Kokain vor dem Essen als Spray- oder Pinselflüssigkeit. Alkoholinjektionen in den Nervuslaryngeus können hier auch versucht werden. Die Inhalationstherapie steht bei mir ganz im Hintergrund. Das tiefere Atmen beim Inhalieren schädigt zu leicht die Lunge. Außerdem wird die Schleimhaut empfindlicher gegen Schwankungen der Lufttemperatur. Inhalationen sind aber oft von Nutzen bei stärkerer Sekretion im Kehlkopf, vor allem dann, wenn das Sekret zäh ist und eintrocknet. Wir bevorzugen in solchen 
Fällen das Einatmen ätherischer Oele mit dem Sängerschen Apparat. (Rp. Guajakol 8,0, Eukalyptol 7,0, Terpineol 6,0, Olei pini pumilion. 5,0, Menthol 4,0, Essentiae syı ingor. 3,0.) Auch Kochsalzwässer sind nützlich. Nach Abschluß einer operativen Therapie hat uns das phenylpropiolsaure Natron, in $1-20$ igen Lösungen irhaliert, oft genützt. Die Schleinhaut schien sich schneller zu erholen und ihr normales Aussehen wiederzugewinnen. $5-10{ }^{\circ}$ ige Nirvaninlösungen inhaliert nützen oft bei Schluckbeschwerden. Man lasse stets mit vorgezogener Zunge oberflächlich einatmen, damit die tieferen Luftwege nicht gereizt werden. Die Inhalation muß der Lokalbehandlung vorangehen.

Ueber die Behandlung der Larynxtuberkulose mit Röntgenstrahlen liegen noch wenige Berichte vor. Uns fehlen darüber Erfahrungen, ebenso über die Jod-Quecksilberbehandlung nach $K o ̈ r n e r$, die in den seltenen Fällen von primärer Tuberkulose des Larynx versucht werden kann.

Mit diesen milden Behandlungsmethoden erreicht man in vielen, leichteren Fällen von Larynxtuberkulose oft schon beste Erfolge. Fache Ulcera granulieren und schließen sich, Infiltrate gehen zurück. Diese Heilerfolge werden nicht in letzter Linie durch Besserung des Lungenleidens und des Allgemeinzustandes bewirkt. Wenn der Organismus instandgesetzt wird und sich fähig zeigt, die Lungentuberkulose zu überwinden, wird er auch mit der Larynxtuberkulose oft ohne akiiveres Vorgehen des Therapeuten fertig. Bei der Beurteilung der Heilwirkung angewandter Mittel soll man stets dieses Faktun voll in Rechnung stellen, um Selbsttäuschungen zu vermeiden. - Viele Fälle von Larynxtuberkulose zeigen eine große Neigung zur Spontanheilung. Diese Tatsache darf uns aber nicht zum therapeutischen Nihilismus bei dieser Krankheit verführen, wie es leider geschehen ist. Sie mahnt uns aber immer wieder, mit aktiverem Vorgehen zunächst für längere Zeit möglichst zurückhaltend $\mathrm{zu}$ sein. Man soll die Fälle erst beobachten. Sie müssen alle vorhin aufgeführten Indikationen voll exfüllen, bevor operative Eingriffe gerechtfertigt sind, die dann sehr segensreich wirken können.

\section{Operative Maßnahmen.}

Wir gehen möglichst radikal vor. Das Kranke soll entfernt werden, möglichst so, daß die Funktion des Organs wenig oder garnicht gestört wird.

Die speziellen Indikationen für unsere Eingriffe richten sich nach der Lokalisation und dem anatomischen Produkt der Krarkheit. Flachere Ulzerationen, die nicht stark granulieren, können mit Aetzungen zur Heilung gebracht werden. Die Milchsäure steht hier im Vordergrunde. Man beginnt mit $25^{\circ}$ iger lösung und steigt langsam mit der Kor zentration bis zur unverdünnten. Wird sie nicht vertragen, was sich oft durch unangenehme Reizerscheinungen äußert, verwenden wir Menthorol (10-15 \%) oder Dianol II und III (Di- und Trilactat), welches Blumenfeld in die Therapie einführte. Vorsichtige Aetzungen mit Acidum trichloraceticum werden auch oft sehr gut vertragen. Die Individualität des Kranken bestimmt in erster Linie das Mittel. Grundsatz ist, die Aetzungen erst zu. wiederholen, wenn der Schorf nach $\mathrm{d} \epsilon \mathrm{m}$ vorangehenden Eing iff sich ganz abgestoßen hat. Stärkere Granulationen werden entweder mit der einfachen Kurette abgeschabt und der Geschwürsgrund dann geätzt, oder man brennt sie mit dem Kauter fort. Der Kauter zerstört dann auch den Grund des Ulcus. Es muß ein starker Brandschorf entstehen. Mit dem Kauter sei man in der Gegend der Stimmbänderansätze vorsichtig, da eine zu feste Narbenbildung hier zu unangerehmen Funktionsstörungen führen kann; auch Ulzerationen der Stimmbänder sind nöglichst durch schorendere Aetzungen zur Vernarbung zu bringen. Der Glühbrenner tritt vor allem bei zerfallenen Infiltraten der Hinterwand in sein Recht. Nicht zerfallene Infiltrate der Taschenbänder, der Arygegend, der a yepiglottischen Falten können durch Stichelungen nach Grünwald sehr gebessert werden. Flache Infiltrate dieser Gegend, der Hinterwand und Stimmbänder, können sich auch nach intramukösen Injektionen von $2 \%$ igen Novokainlösungen nach Spiess zurückbilden. Werden sie tumorig, dann trägt man sie entweder mit der Doppelkurette ab und brennt den Grund oder, wenn sie zu fassen sind, entfernt man sie mit der Glühschlinge, die auch bei manchen tumorigen Verdickungen der Stimmbänder und Hinterwand angezeigt ist. Bei Ulzerationen und Wucherungen in subglottischen Raum sei man mit der Anwendung des Glühbrenners recht vorsichtig. Es entstehen dort leicht heftige Oedeme, die zur Suffokation führen.

Besteht eine isolierte Epiglottistuberkulose ohne oder nit nur geringen tuberkulösen Veränderungen im inneren Larynx und mit Freisein der aryepiglottischen Falten, dann ist die Anıputation des KehIdeckels angezeigt, die beste Erfolge gibt. Man kann sie blutig mit schneidendon Zangen nach Schmidt und Jurasz oder mit der Guillotine von A le xander ausführen, mit der kalten Schlinge nach Gerber oder mit der Glühschlinge. Die Kranken schlucken nach Entfernung des Kehldeckels meistens sofort ohne nennenswerte Beschwerden.

Ueber die Laryngofissur, die totale Entfernung des tuberkulösen Larynx nach GIuck, die kurative Tracheotomie fehlen mir eigene Erfahrungen. Solche eingreifende Operationen sind nur in den seltensten Föllen bei völlig latenter Lungentuberkulose, bestem Allgemeinbefinden und schwersten unheilbaren Larynxprozessen angezeigt, Bedingungen, die nicht häufig erfüllt werden. Die bis jetzt vorliegenden Erfolge dieser Verfahren sind nicht sehr ermutigend. - Sie sehen, es können ja nach dem Falle die verschiedensten operativen Verfahren bei der Larynxtuberkulose zur Anwendung kommen. Es ist falsch, sich nur auf Aetzungen mit Chemikalien zu beschränken, ebenso falsch, nur die Kuretten zu benutzen oder nur den Glühbrenner.

Je nach dem Falle kombiniere man alle Verfahren. Wir müssen allerdings bekennen, daß auch bei uns der Glühbrenner die Kurettage etwas in den Hintergrund gedrängt lat.

Man kann mit dem Brenner besser lokalisieren. Der Eingriff ist für den Kranken schonender. Daß alle genannten Eingriffe nur nach bester Anästhesierung zu erfolgen haben, ist selbstverst ändlich. Auch die ätzenden Chemikalien sind streng lokal anzuwenden. Nach erfolgtem Eingriff tritt die konservative Behandlung wieder in ihr Recht.

Nun möchte ich Ihnen noch kurz unsere Erfolge mitteilen, die wir unter Befolgen genannter Indikationen für unser therapeutisches Handeln erzielten. Ich habe die Fälle tabellarisch geordnet, sie insgesamt betrachtet und in geschlossene und offene Tuberkulosen, in fiebernde und nichtfiebernde Kranke gesondert. Wir fanden, daß wir in $58 \%$ der Fälle beste und gute Heilerfolge erzielten; daß die Erfolge hervonarend gut waren bei geschlossenen und fieberlos verlaufenden Tuberkulosen. Das sind die Fälle, die vor allem für die Volksheilstätten in Frage kommen. Ich wiederhole hier nochmals die Wichtigkeit der Frühdiagnose. - Auf nebenstehender Tabelle sind die Entlassungserfolge verzeichnet. Sie werden sofort nach den Dauererfolgen fragen. Eine Statistik an der Hand einer Enquete hat hier nicht viel Wert, da der Larynx der Entlassenen entweder von uns selbst oder von Spezialisten genau hätte nachkontrolliert werden müssen, was nicht immer möglich ist. Wir sind trotzdem bemüht, genaue Nachforschungen nach den frühər von uns behandelten Larynxphthisikern anzustellen, und hoffen später über das Resultat berichten zu können. Bei einer großen Reihe von Fällen konnten wir eine Heilung des Larynx, die schon jahrelang standhielt, feststellen.

Gestatten Sie mir noch zum Schluß einige Bemerkungen über den Nutzen der spezifischen Therapie bei der Larynxtuberkulose.

Es gibt einige Therapeuten, die gute Heilungen der Larynxtuberkulose nach Anwendung von Tuberkulinpräparaten sahen. Die neisten Laryngologen haben keinen Nutzen vom Tuberkulin gehabt, viele Schädigungen und Ausbreitungen der Krankheit im Kehlkopf nach seiner Anwendung gesehen. Wie widersprechend dic Anschauungen hier lauten können, zeigt folgendes Beispiel. Bandelier (4) sah gute Heilungen nach Tuberkulingebrauch, Clarus (5), der neben Bandelier an Weickers Krankenheim arbeitete, hält die Tuberkulinbehandlung für 
Statistik über Kurerfolge bei 416 Fällen von Larynxtuberkulose. Mittlere Kurdauer 140 Tage.

\begin{tabular}{|c|c|c|c|c|c|c|}
\hline \multirow[b]{2}{*}{ Stadien } & \multirow[b]{2}{*}{ Zahl } & \multirow{2}{*}{$\begin{array}{l}\text { relative } \\
\text { Heilung }\end{array}$} & \multicolumn{2}{|c|}{ Erfolge } & \multirow{2}{*}{$\begin{array}{c}\text { gleich } \\
\text { geblieben }\end{array}$} & \multirow[b]{2}{*}{ gestorben } \\
\hline & & & $\begin{array}{c}\text { gut } \\
\text { gebessert }\end{array}$ & gebessert & & \\
\hline 1 & 45 & 23 & 16 & 2 & 4 & - \\
\hline 2 & $\begin{array}{l}10,8 \% \\
196 \\
47,1 \%\end{array}$ & 57 & 77 & 26 & 26 & 10 \\
\hline 3 & $\begin{array}{r}175 \\
42,1 \% \\
\end{array}$ & 20 & 48 & 23 & 65 & 19 \\
\hline Summa & 416 & $\begin{array}{l}100 \\
24 \%\end{array}$ & $\begin{array}{c}141 \\
33,9 \%\end{array}$ & $\begin{array}{c}51 \\
12,2 \%\end{array}$ & $\begin{array}{c}95 \\
22,6 \%\end{array}$ & $\begin{array}{c}29 \\
7,3 \%\end{array}$ \\
\hline
\end{tabular}

Fiebernde.

\begin{tabular}{c||cc||c|c||cc}
\hline 1 & 19 & 5 & 10 & 2 & 2 & - \\
2 & 135 & 29 & 51 & 18 & 29 & 8 \\
\hline 3 & 154 & 13 & 35 & 22 & 65 & 19 \\
\hline Summa & 308 & 47 & 96 & 42 & 96 & 27 \\
& $73,8 \%$ & $15,2 \%$ & $31,1 \%$ & $13,6 \%$ & $31,1 \%$ & $8,7 \%$
\end{tabular}

Fieberlose.

\begin{tabular}{c||c|c||c|c||c|c}
\hline 1 & 26 & 18 & 7 & - & 1 & - \\
3 & 61 & 28 & 26 & 5 & 2 & $=$ \\
\hline Summa & 108 & 7 & 13 & 1 & - & - \\
& $26,2 \%$ & $48,1 \%$ & $42,5 \%$ & $5,5 \%$ & $2,7 \%$ & -
\end{tabular}

Oeschlossene Tuberkulosen.

\begin{tabular}{|c|c|c|c|c|c|c|}
\hline $\begin{array}{l}1 \\
2 \\
3 \\
\end{array}$ & $\begin{array}{r}15 \\
90 \\
8 \\
\end{array}$ & $\begin{array}{r}11 \\
15 \\
3 \\
\end{array}$ & $i$ & $\bar{z}$ & $\begin{array}{l}3 \\
- \\
-\end{array}$ & $\bar{z}$ \\
\hline Summa & $\begin{array}{c}43 \\
10,4 \%\end{array}$ & $\begin{array}{c}29 \\
67,4 \%\end{array}$ & $\begin{array}{c}11 \\
25,6 \%\end{array}$ & $\overline{-}$ & $\begin{array}{l}3 \\
7 \%\end{array}$ & - \\
\hline \multicolumn{7}{|c|}{ Offene Tuberkulosen. } \\
\hline $\begin{array}{l}1 \\
2 \\
3 \\
\end{array}$ & $\begin{array}{r}30 \\
106 \\
167 \\
\end{array}$ & $\begin{array}{l}12 \\
42 \\
17 \\
\end{array}$ & $\begin{array}{r}15 \\
72 \\
43 \\
\end{array}$ & $\begin{array}{r}2 \\
26 \\
23 \\
\end{array}$ & $\begin{array}{r}1 \\
26 \\
65 \\
\end{array}$ & $\begin{array}{l}\overrightarrow{10} \\
19\end{array}$ \\
\hline Summa & $\begin{array}{c}37,3 \\
87,6 b\end{array}$ & $\begin{array}{l}71 \\
19 \%\end{array}$ & $\begin{array}{l}130 \\
35 \%\end{array}$ & $\begin{array}{c}51 \\
13,7{ }^{\prime}\end{array}$ & $\begin{array}{c}92 \\
24,6 \%\end{array}$ & $\begin{array}{r}29 \\
7,7 \%\end{array}$ \\
\hline
\end{tabular}

nicht ausreichend für die Kehlkopftuberkulose. Wir müssen uns ihm anschließen. Es ist ein Fehler, sich bei der Therapie der Larynxtuberkulose nur dem Tuberkulin auzuvertrauen. Wir haben an 29 Fällen den Eindruck gewonnen, daß die spezifische Therapie kaum Nutzen stiftet, in manchen unserer Fälle vielleicht sogar an der Ausbreitung des Prozesses im Kehlkopf, dem Eintreten von Zerfall der Infiltrate schuld war. Man kann sie, bei vorsichtiger Dosierung des Präparates, nur ein Unterstützungsmittel nennen. Unsere spezifisich behandelten Fälle wurden nach den Grundsätzen gespritzt, die ich im vorigen Jahre auf unserer Versammlung in Cassel entwickelte (8).

M. H.! Gehen Sie also zielbewußt bəi der Behandlung der Larynxtuberkulose vor, berücksichtigen Sie in erster Linie den Allgemeinzustand Threr Kranken, stellen Sie strengste Indikationen für eingreifendere Maßnahmen, dann werden Sie bei manchen Fällen von Larynxtuberkulose die schönsten andauernden Erfolge sehen.

Zusammenfassung. 1. Für eine erfolgreiche Behandlung der Larynxtuberkulose ist die Frühdiagnose sehr wichtig, die fast sicher durch lokale Inspektion gestellt werden kann. Jeder lungenkranke ist genau im Larynx zu untersuchen.

2. Die Larynxtuberkulose ist am erfolgreichsten in der geschlossenen Anstalt zu bahandeln. Die Volksheilstätten haben sich der noch heilbaren Larynxphthisiker anzunehmen.

3. Der Kern der Therapie ist die Allgemeinbehandlung. Nur Besserung und Heilung der Lungen verspricht für den Larynx einen Erfolg. Jeder noch fiebernde oder zum Fieber neigende Kranke muß konservativ bohandelt werden, ferner Kranke, bei denen Komplikationen vorliegen, die eine Neigung zur Propazation des Lungenprozesses befürchten lassen.

4. Bei geeigneten Kranken erreicht man durch zielbewußtes chirurgisches Eingreifen gute Erfolge.

5. Die Tuberkulinbehandlung ist bei Larynxtuberkulösen nur mit größter Reserve und Vorsicht zu verwerten. Sie ersetzt nie die Lokalbehandlung.

Benutzte Literatur: 1. Da Gradi, Deutsche medizin sche Wochenschrift No. 22. 12. Juni 1910. - 2. Pott enger, California State, Journal of Med., Oktober 1903 . - 3. Besold und Gidionsen, Pathologie und Therapie der Kehlkopftuberkulose. Berlin 1907. - 4 Bandelier, Beiträge zur Klinik der Tuberkulose, Bd. 15, H. 1. 5. Clarus, ibidem, Bd. 15, H. 2. - 6. G. Schröder und Nägeis bach, Württem. bergisches medizinisches Korrespondenzblatt 1902. - 7. Burger, Zeitschrift für kli: nische Medizin, Bd. 62. - 8. G. Schröder, Beiträge zur Klinik der Tuberkulose,
Bd. 14, H. 4. - 9. Weitere ausfūhrliche Literaturangaben findet man in $\mathrm{H}$ e y m a n ns Handbuch der Laryngologie und Rhinologie und im Handbuch der Therapie der Lungenschwindsucht von G. S chröder und F. Blu menfeld. 